\title{
A non-negative representation learning algorithm for selecting neighbors
}

\author{
Lili Li ${ }^{1} \cdot$ Jiancheng $\mathrm{Lv}^{1} \cdot$ Zhang $\mathrm{Yi}^{1}$
}

Received: 8 August 2014 / Accepted: 22 April 2015 / Published online: 7 May 2015

(C) The Author(s) 2015

\begin{abstract}
A crucial step in many manifold clustering and embedding algorithms involves selecting the neighbors of a data point. In this paper, a non-negative representation learning algorithm is proposed to select the neighbors of a data point, so that they are almost always lying in the tangent space of the manifold at the given query point. This is very important to multi-manifold clustering and manifold embedding. Convex geometry theory states that a data point on a face of a convex polytope can be a convex combination of the vertices of the polytope, and that the non-zero combination scalars correspond to the vertices of the face the point lies on. The intent of this paper is to use points that that are near to a point as the vertices of a convex polytope, and select the vertices on the same face as this point to be the neighbors. Clearly, the point can be a convex combination of its neighbors. We can observe that these neighbors almost always lie in the tangent space of the manifold at the query point and can preserve the local manifold structure well. Based on this basic idea, we construct an objective function. Moreover, an augmented Lagrange multipliers method is proposed for solving it to derive a non-negative representation, and then the neighbors of a data point can be obtained. The result of our neighbor selection algorithm can be used by other clustering methods such as LEM or manifold embedding methods such as LLE. We demonstrate the effectiveness and efficiency of the proposed method through experiments on synthetic data and a real-world problem.
\end{abstract}

Keywords Convex combination · ALM method · Manifold embedding · Multiple manifolds clustering $\cdot$ Neighbors selection

Editor: Xiaoli Z. Fern.

Jiancheng Lv

lvjiancheng@scu.edu.cn

Lili Li

lilili_cd@cuit.edu.cn

Zhang Yi

zyiscu@gmail.com

1 Machine Intelligence Laboratory, College of Computer Science, Sichuan University, Chengdu 610065, People's Republic of China 


\section{Introduction}

In many areas of computer science the data typically lies on a low-dimensional manifold embedded in an ambient space of high dimensions. Moreover, real-world data are often represented on multiple manifolds. Examples of this can be usually found in machine learning, signal and image processing, pattern recognition, computer vision and information retrieval. Thus, to find a low-dimensional embedding of the data, one needs to first cluster the data according to the underlying manifolds and then find a low-dimensional representation for the data in each cluster (Elhamifar and Vidal 2011). Most manifold clustering and dimensionality reduction methods depend on the eigenvectors of a matrix related to the learned weights between pairs of points. Neighbor selection plays a crucial role in these methods.

Many manifold clustering and embedding algorithms have been proposed. Linear model methods, such as low-rank representation (LRR) (Liu et al. 2010), sparse subspace clustering (SSC) (Elhamifar and Vidal 2009), principal component analysis (PCA) (Vidal et al. 2005; Lv et al. 2010; Cheng et al. 2010; Lv et al. 2009) and expectation maximization (EM) method (Tipping and Bishop 1999), are only used to process linear data. Nonlinear methods such as locally linear embedding (LLE) (Tenenbaum et al. 2000) are employed for manifold embedding and can be extended to perform clustering (Polito and Perona 2002; Goh and Vidal 2007; Goh and Vidal (2008)). Traditional k-nearest neighbor (kNN) or improved kNN (Ferrandiz and Boullé 2010; JIANG et al. 2009) are used for selecting neighbors in these nonlinear methods. It is usually assumed that, to learn a low-dimensional representation with the intrinsic structure, there exists an appropriately sized neighborhood that captures the local structure of a manifold while excluding points from other manifolds. However, it is not easy to determine the size of this neighborhood, especially when different manifolds are very close to each other.

Recently, an algorithm called sparse manifold clustering and embedding (SMCE) (Elhamifar and Vidal 2011) was proposed to automatically find the neighbors for manifold clustering and embedding. This method has been used in manifold alignment ( $\mathrm{Li}$ et al. 2013). SMCE is based on the assumption that for all $i$ there exists $\epsilon>0$ such that the nonzero entries of the sparsest solution of an optimization problem correspond to the $d_{l}+1$ neighbors of $x_{i}$ from $M_{l}$, where $N$ data points $\left\{x_{i} \in R^{D}\right\}_{i=1}^{N}$ lie on $n$ different manifolds $\left\{M_{l}\right\}_{l=1}^{n}$ of intrinsic dimensions $\left\{d_{l}\right\}_{l=1}^{n}$. However, it is not easy to control the parameter $\epsilon>0$ so that this assumption holds. Moreover, the sparest solution of the optimization problem is derived by solving a $l_{1}$ norm, but determining the number of nonzero entries of the solution is challenging. In addition, the objective function of SMCE does not guarantee the non-negativity of the nonzero entries of the solution as it has assumed.

In this paper, we propose a non-negative representation learning algorithm to select the neighbors of a data point based on convex polytope theory, in which a data point on a face of a convex polytope can be a convex combination of vertices of this face. First, we prove that if point $x_{i}$ is on a face, the non-zero convex combination scalars correspond to the vertices of the face $x_{i}$ lies on. To use this theorem to select the points from the tangent space of the manifold at $x_{i}$ to be the neighbors, we design two cooperative mechanisms for placing $x_{i}$ on a face of a convex polytope, but some other points lying in this tangent space to be the vertices of this face. Second, an objective function is constructed based on this idea. Specially, we use $\|\cdot\|_{1}$ norm to obtain a non-negative representation. Finally, we find a non-negative representation using the augmented Lagrange multipliers (ALM) method to obtain the neighbors of a data point. The neighbors selected by our method almost always come from this tangent space and preserve the local geometry structure well. Therefor, the result of the neighbor selection 
can be used for manifold embedding and multiple manifold clustering. A large number of experiments illustrate the effectiveness and efficiency of this method.

The remainder of this paper is organized as follows. Two theorems used in our proposed method are presented in Sect. 2. A new method for neighbor selection from the tangent space at the given query point is sketched in Sect. 3, and the discussion of experiments and conclusions are described in Sects. 4 and 5 respectively.

\section{Preliminaries}

In this section we present two theorems that provide the theoretical basis for our method. First, we list three definitions for convex polytopes that will be used in our theorems.

Definition 1 A vector $x$ is called a convex combination of points $x_{1}, \ldots, x_{N}$ in $\mathcal{R}^{n}$ if it has the form

$$
x=\sum_{i=1}^{N} \lambda_{i} x_{i} \quad \text { where } \quad \lambda_{i} \geq 0 \text { and } \sum_{i=1}^{N} \lambda_{i}=1 .
$$

In Problem (1), $\lambda_{i}$ is the weight of $x_{i}$. If $\lambda_{l}>0(1 \leq l \leq N)$, we say that point $x_{l}$ has a positive contribution to $\mathrm{x}$; if $\lambda_{l}=0(1 \leq l \leq N)$, we say that point $x_{l}$ has a zero contribution to $\mathrm{x}$.

Definition 2 (Henk et al. 1997) Given a polytope $P \subseteq \mathcal{R}^{n}$, the function

$$
h(P, \cdot): \mathcal{R}^{n} \longrightarrow R, h(P, x):=\sup \{\langle x, y\rangle \mid y \in P\},
$$

is the support function, where $x, y$ denotes the inner product on $\mathcal{R}^{n}$. (Since $P$ is compact one may replace sup by max.) For $v \in \mathcal{R}^{n} \backslash\{0\}$ the hyperplane $H(P, v):=\left\{x \in \mathcal{R}^{n} \mid\langle x, v\rangle=\right.$ $h(P, v)\}$ is the supporting hyperplane of $P$ with outer normal vector $v$. The intersection of $P$ with a supporting hyperplane $H(P, v)$ is called a (nontrivial) face, or more precisely a $k$-face if the dimension of $\operatorname{aff}(P \cap H(P, v))$ is $k$. Each face itself is a polytope.

Definition 3 (Grunbaum et al. 1967) Let $K$ be a convex subset of $\mathcal{R}^{n}$. A point $x \in K$ is an extreme point of $K$ provided $y, z \in K, 0<\alpha<1$, and $x=\alpha y+(1-\alpha) z$ imply $x=y=z$. The set of all extreme points of $K$ is denoted by ext $K$. Clearly, if $x \in \operatorname{ext} K$ then $x \notin \operatorname{conv}(K-\{x\})$. For a polytope (or polyhedral set) $K$, it is customary to call the points of ext $K$ vertices, and to denote their totality by vert $K$.

In Problem (1), suppose that points $x_{1}, \ldots, x_{N}$ are the vertices of polytope $P(P \subseteq$ $\left.\mathcal{R}^{n}\right)$. If $H_{1}$ is a supporting hyperplane of $P$ with $F_{1}=H_{1} \cap P$, then $F_{1}$ is a face of polytope $P$ and vert $F_{1} \subseteq \operatorname{vert} P$. Let vert $F_{1}=\left\{x_{f_{1}}, x_{f_{2}}, \ldots, x_{f_{k_{1}}}\right\}$ and vert $P-$ vert $F_{1}=\left\{x_{f_{1}^{*}}, x_{f_{2}^{*}}, \ldots, x_{f_{k_{2}}^{*}}\right\}$ (where $\left.k_{1}+k_{2}=N\right)$, then vert $P=$ $\left\{x_{f_{1}}, x_{f_{2}}, \ldots, x_{f_{k_{1}}}, x_{f_{1}^{*}}, x_{f_{2}^{*}}, \ldots, x_{f_{k_{2}}^{*}}\right\}=\left\{x_{1}, x_{2}, \ldots, x_{N}\right\}$. If a point $x \in P$, then $x$ can be a convex combination of all the vertices of $P$, that is,

$$
x=\sum_{i=1}^{k_{1}} \lambda_{f_{i}} x_{f_{i}}+\sum_{i=1}^{k_{2}} \lambda_{f_{i}^{*}} x_{f_{i}^{*}}=\sum_{i=1}^{N} \lambda_{i} x_{i},
$$


Fig. 1 Data point $x_{1}$ lies in $\operatorname{conv}\left(\left\{V_{1}, V_{2}, V_{3}\right\}\right)$ and $x_{2}$ lies in $\operatorname{conv}\left(\left\{V_{1}, V_{2}\right\}\right)$. In any convex combination of all vertices, $V_{4}$ has a zero contribution to $x_{1} . V_{3}$ and $V_{4}$ have zero contributions to $x_{2}$

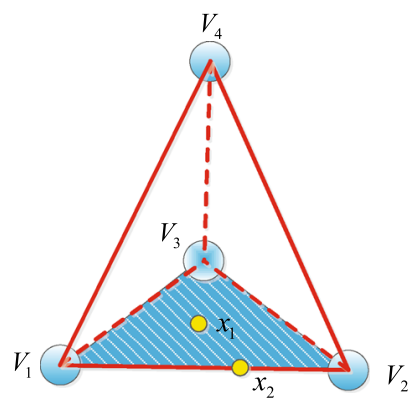

where $\sum_{i=1}^{k_{1}} \lambda_{f_{i}}+\sum_{i=1}^{k_{2}} \lambda_{f_{i}^{*}}=\sum_{i=1}^{N} \lambda_{i}=1, \lambda_{f_{i}} \geq 0\left(i=1, \ldots, k_{1}\right), \lambda_{f_{i}^{*}} \geq 0\left(i=1, \ldots, k_{2}\right)$, and $\lambda_{i} \geq 0(i=1, \ldots, N)$.

For a point $x$ on a face of a convex polytope, all vertices that are not on this face must have zero contributions to $x$ in the convex combination in Problem (2). Here we shall prove the following:

Theorem 1 Let a point $x \in P$ in Problem (2). If $x \in F_{1}$ and if $\lambda_{l}>0$ (or $\lambda_{l} \neq 0$ ), then $x_{l} \in \operatorname{vert} F_{1}(1 \leq l \leq N)$.

Proof If $x \in F_{1}$, then $x$ can be a convex combination of all the vertices of $F_{1}$, that is,

$$
x=\sum_{i=1}^{k_{1}} \lambda_{f_{i}} x_{f_{i}} \text { where } \sum_{i=1}^{k_{1}} \lambda_{f_{i}}=1 \text { and } \lambda_{f_{i}} \geq 0 .
$$

According to (2), it must hold that $\lambda_{f_{i}^{*}}=0\left(i=1,2, \ldots, k_{2}\right)$. Then, if $\lambda_{l}>0\left(\right.$ or $\left.\lambda_{l} \neq 0\right)$ in (2), it must hold that $x_{l} \in \operatorname{vert} F_{1}(1 \leq l \leq N)$. The proof is complete.

Theorem 1 states that every vertex $x_{f_{i}^{*}}\left(i=1, \ldots, k_{2}\right)$ that does not lie on face $F_{1}$ must have zero contribution to point $x$. For example, in Fig. 1 data point $x_{1}$ lies in $\operatorname{con} v\left(\left\{V_{1}, V_{2}, V_{3}\right\}\right)$, which is a face of $\operatorname{conv}\left(\left\{V_{1}, V_{2}, V_{3}, V 4\right\}\right)$. In any convex combination of all the vertices, $V_{4}$ always has a zero contribution to $x_{1}$. Data point $x_{2}$ lies in $\operatorname{conv}\left(\left\{V_{1}, V_{2}\right\}\right)$, which is a face of $\operatorname{conv}\left(\left\{V_{1}, V_{2}, V_{3}, V_{4}\right\}\right)$. In any convex combination of all the vertices, $V_{3}$ and $V_{4}$ always have zero contributions to $x_{2}$.

On the contrary, if $x$ lies on face $F_{1}$, the vertex that has a positive contribution to $x$ in the convex combination in Problem (2) must lie on this face. Then, if all vertices of $F_{1}$ are from the tangent space of the manifold at $x$, from Theorem 1 we may select the points with nonzero weights in Problem (2) to be it's neighbors. Naturally, the neighbors selected by this method must be in this tangent space. In this paper, two cooperative mechanisms are designed to construct such a convex polytope and a method based on this theorem for selecting the neighbors is proposed.

The representation of $x$ in Problem (2) can be obtained by solving the following optimization problem:

$$
\left\{\begin{array}{l}
\min _{\lambda}\left\|x-\sum_{i=1}^{N} \lambda_{i} x_{i}\right\|_{2} \\
\text { subject to } \mathbf{1}^{T} \lambda=1 \text { and } \lambda_{i} \geq 0,
\end{array}\right.
$$


where $\mathbf{1}^{T} \triangleq[1,1, \ldots, 1] \in \mathcal{R}^{N}$ and $\lambda^{\top} \triangleq\left[\lambda_{1}, \lambda_{2}, \ldots, \lambda_{N}\right] \in \mathcal{R}^{N}$. Theorem 2 states that the non-negative condition $\lambda_{i} \geq 0$ can be replaced by $\|\lambda\|_{1} \leq 1$ under certain condition, where $\|\lambda\|_{1}=\sum_{i=1}^{N}\left|\lambda_{i}\right|$.

Theorem 2 Let $\lambda \in \mathcal{R}^{N}$ be a vector. If $\mathbf{1}^{T} \lambda=1$ and $\|\lambda\|_{1} \leq 1$, then $\lambda_{i} \geq 0(i=1,2, \ldots, N)$.

Proof Suppose $\exists \lambda_{l}<0(1 \leq l \leq N)$ and $\forall \lambda_{j} \geq 0(j=1,2, \ldots, N$ and $j \neq l)$. It holds that

$$
\|\lambda\|_{1}=\sum_{i=1}^{N}\left|\lambda_{i}\right|=\left|\lambda_{l}\right|+\sum_{j \neq l}\left|\lambda_{j}\right|=-\lambda_{l}+\sum_{j \neq l} \lambda_{j} .
$$

Since

$$
\mathbf{1}^{T} \lambda=\sum_{i=1}^{N} \lambda_{i}=\sum_{j \neq l} \lambda_{j}+\lambda_{l}=1,
$$

Eq. (4) can be rewritten as

$$
\|\lambda\|_{1}=-\lambda_{l}+\sum_{j \neq l} \lambda_{j}=-\lambda_{l}+1-\lambda_{l}=1-2 \lambda_{l}>1 .
$$

This means that, under the condition $\mathbf{1}^{T} \lambda=1$, if there exits a negative element in the vector $\lambda$, then $\|\lambda\|_{1}>1$, which is a contradict with the condition $\|\lambda\|_{1} \leq 1$. So, if $\mathbf{1}^{T} \lambda=1$ and $\|\lambda\|_{1} \leq 1$, every element of $\lambda$ is non-negative. The proof is complete.

From Theorem 2, we know that the non-negative condition $\lambda_{i} \geq 0$ can be replaced by $\|\lambda\|_{1} \leq 1$ under the condition $\mathbf{1}^{T} \lambda=1$. Then, the optimization problem in (3) can be rewritten as

$$
\left\{\begin{array}{l}
\min _{\lambda}\left\|x-\sum_{i=1}^{N} \lambda_{i} x_{i}\right\|_{2} \\
\text { subject to } \mathbf{1}^{T} \lambda=1 \text { and }\|\lambda\|_{1} \leq 1 .
\end{array}\right.
$$

Another optimization problem that is related to the optimization problem in (5) by the method of Lagrange multipliers, is

$$
\left\{\begin{array}{l}
\min _{\lambda} \gamma\left(\|\lambda\|_{1}-1\right)+\frac{1}{2}\left\|x-\sum_{i=1}^{N} \lambda_{i} x_{i}\right\|_{2}^{2} \\
\text { subject to } \mathbf{1}^{T} \lambda=1 .
\end{array}\right.
$$

The optimization problems in (5) and (6) can both be used to solve Problem (2). In this paper, we use $\|.\|_{1}$ norm to obtain the representation of $x$.

\section{Selecting neighbors using a non-negative representation learning algorithm}

Theorem 1 states that some of the vertices on the same face as $x$ can be exactly selected by the optimization problem in (3). If the vertices of this face are from the tangent space of the manifold at the given query point, we can use the solution of this optimization problem for 
effective clustering. In Subsection 3.1, we describe the detailed mechanisms that only points from this tangent space are placed on a face. From Theorem 2 we know that the non-negative condition can be replaced by $\|.\|_{1}$ norm under certain condition. This method is used in our optimization problem in Subsection 3.2. In Subsection 3.3, we propose a new method of selecting $k$ neighbors and give an ALM algorithm for solving our optimization problem.

\subsection{Motivation for non-negative representation-based neighbor selection}

Consider a set of data vectors $X=\left[x_{1}, x_{2}, \ldots, x_{N}\right] \in \mathcal{R}^{D \times N}$ (each column is a point in $\mathcal{R}^{D}$ ), lying on a union of $k$ different manifolds $\left\{M_{l}\right\}_{l=1}^{k}$. Let $\left\{d_{l}\right\}_{l=1}^{k}$ be the intrinsic dimensions of the $k$ manifolds. We assume that a manifold is locally linear in the neighborhood. Suppose that tangent space (or affine space) $T_{x_{i}}$ of $M_{l}$ at $x_{i}$ is $d_{l}$ dimensional (where point $x_{i}$ lies on manifold $M_{l}$ ). Let $N_{x_{i}} \subseteq\left\{x_{j}\right\}_{j \neq i}$ be a set of points which are among the nearer to $x_{i}$ from $M_{l}$ and lie in space $T_{x_{i}}$.

If a convex polyhedron can be constructed that the vertices are all points (except for $x_{i}$ ) from different manifolds and $x_{i}$ lies on one of it's faces, and if the points lying in tangent space $T_{x_{i}}$ are the vertices of this face, based on Theorem 1, the points with nonzero weights in Problem (3) must come from tangent space $T_{x_{i}}$. Thus, these points can be used to be neighbors. Generally, it's practically impossible that all points in $X$ correspond exactly with the vertices of a convex polyhedron.

To construct a convex polyhedron, we normalize the data vectors $\left\{x_{j}-x_{i}\right\}_{j \neq i}$ and let

$$
x_{j^{\prime}} \triangleq \frac{x_{j}-x_{i}}{\left\|x_{j}-x_{i}\right\|_{2}}+x_{i}
$$

where $\left\|x_{j^{\prime}}-x_{i}\right\|_{2}=1$. We call $x_{j^{\prime}}$ the normalized point of $x_{j}$. The points in set $\left\{x_{j^{\prime}}\right\}_{j \neq i}$ are distributed on the surface of the unit ball centered at $x_{i}$. Obviously, these points are the vertices of a convex polyhedron (denoted by $P$ ). Theorem 3 states that if an original point $x_{j}(j \neq i)$ lies in tangent space $T_{x_{i}}$, it's normalized point $x_{j^{\prime}}$ lie in this space. We know that the points in set $N_{x_{i}}$ lie in $T_{x_{i}}$. Let $N_{x_{i}}^{\prime} \subseteq\left\{x_{j^{\prime}}\right\}_{j \neq i}$ be a set of normalized points of $N_{x_{i}}$. Then, the points in set $N_{x_{i}}^{\prime}$ are in tangent space $T_{x_{i}}$.

Theorem 3 Suppose that $T_{x_{i}}$ is an affine space (Gallier 2011) that allows for a free transitive action of a vector space $V$. If $x_{j} \in T_{x_{i}}$ then $x_{j^{\prime}} \in T_{x_{i}}$. If $x_{j} \notin T_{x_{i}}$ then $x_{j^{\prime}} \notin T_{x_{i}}$.

Proof It holds that $x_{i} \in T_{x_{i}}$. If $\exists x_{j} \in T_{x_{i}}(j \neq i)$, then $\frac{x_{j}-x_{i}}{\left\|x_{j}-x_{i}\right\|_{2}} \in V$, and then $x_{j^{\prime}} \in T_{x_{i}}$. If $\exists x_{j} \notin T_{x_{i}}(j \neq i)$, then $\frac{x_{j}-x_{i}}{\left\|x_{j}-x_{i}\right\|_{2}} \notin V$, and then $x_{j^{\prime}} \notin T_{x_{i}}$. The proof is complete.

If the vector $x_{i}$ is a convex combination of set $\left\{x_{j^{\prime}}\right\}_{j \neq i}$, it can be formulated as:

$$
\begin{gathered}
x_{i}=\sum_{j=1}^{N-1} c_{i j} x_{j^{\prime}}=\sum_{j=1}^{N-1} c_{i j}\left(\frac{x_{j}-x_{i}}{\left\|x_{j}-x_{i}\right\|_{2}}\right)+x_{i}, \\
\text { where } c_{i j} \geq 0 \text { and } \sum_{j=1}^{N-1} c_{i j}=1 .
\end{gathered}
$$

Let

$$
X_{i} \triangleq\left[\frac{x_{1}-x_{i}}{\left\|x_{1}-x_{i}\right\|_{2}} \cdots \frac{x_{N}-x_{i}}{\left\|x_{N}-x_{i}\right\|_{2}}\right] \in \mathcal{R}^{D \times N-1}
$$




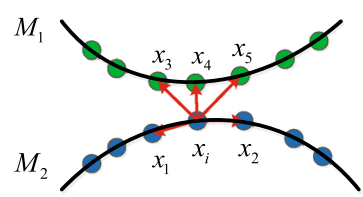

(a)

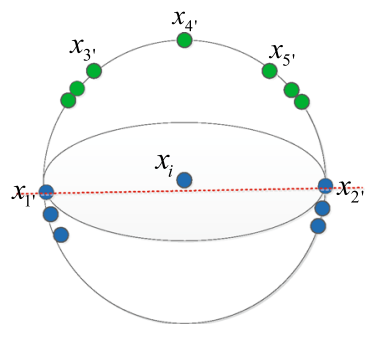

(b)

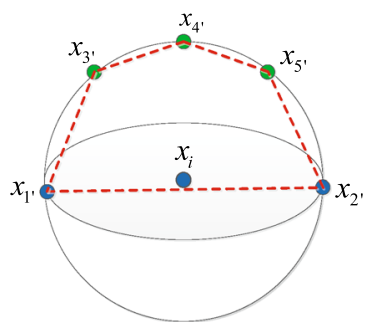

(c)

Fig. 2 Illustrate two cooperative mechanisms for placing the points from the tangent space of the manifold at the given query point on a face of a convex polyhedron. a An example where $x_{i}$ and it's nearest points (belong two different manifolds) are labeled. b Illustrate the mechanism for forming a convex polyhedra: the normalized points in set $\left\{x_{j^{\prime}}\right\}_{j \neq i}$ are distributed on the surface of the unit ball centered at $x_{i}$ and naturally form the vertices of a convex polyhedra. c Illustrate the mechanism for placing the points from tangent space $T_{x_{i}}$ on a face of a convex polyhedra: the farthest points (which are not labeled) are removed, and then the convex combination of points $x_{1^{\prime}}, x_{2^{\prime}}$ is a face of the convex polyhedra and $x_{i}$ lies on this face

and

$$
c_{i}^{\top} \triangleq\left[c_{i 1}, c_{i 2}, \ldots, c_{i N-1}\right] \in \mathcal{R}^{1 \times N-1},
$$

Problem (8) can be formulated as:

$$
X_{i} c_{i}=0 \text { where } c_{i j} \geq 0 \text { and } \mathbf{1}^{T} c_{i}=1 .
$$

Actually, $x_{i}$ can't exactly lie on a face of convex polyhedron $P$. To obtain a new convex polyhedron from $P$, where $x_{i}$ lies on one of it's faces and some points lying in tangent space $T_{x_{i}}$ are the vertices of this face, we instead exploit a simple observation: if the furthest points are removed from $P$, and if $x_{i}$ is a convex combination of the other vertices in this convex polyhedron, the points in set $N_{x_{i}}^{\prime}$ are more likely to be on the same face as $x_{i}$. Note that each point in $N_{x_{i}}^{\prime}$ corresponds to an original data point in $N_{x_{i}}$, which are in tangent space $T_{x_{i}}$. Thus, the points corresponding to the nonzero entries of the solution $c_{i}$ in Problem (9) must lie in this tangent space. The non-negative representation $c_{i}$ can be used for effective clustering.

Two manifolds from Elhamifar and Vidal (2011) are shown in Fig. 2a. These examples are used to illustrate two cooperative mechanisms for forming a convex polyhedron, at the same time, placing $x_{i}$ on a face of this convex polyhedron and placing some points from tangent space $T_{x_{i}}$ on the vertices of this face. Assume that points $x_{1}, x_{2}, x_{3}, x_{4}$ and $x_{5}$ are close to $x_{i}$. Among them, only $x_{1}$ and $x_{2}$ come from manifold $M_{2}$ and are lying in the tangent space of $M_{2}$ at $x_{i}$. After normalizing, the points in set $\left\{x_{j^{\prime}}\right\}_{j \neq i}$ are distributed on the surface of the unit ball centered at $x_{i}$ and naturally form the vertices of a convex polyhedra, as illustrated in Fig. 2b. One can see that $x_{1^{\prime}}$ and $x_{2^{\prime}}$ still lie in tangent space $T_{x_{i}}$, and the other normalized points $\left(x_{3^{\prime}}, x_{4^{\prime}}\right.$ and $\left.x_{5^{\prime}}\right)$ have moved away from this space. However, $x_{i}$ is not on a face. If the furthest points (which are not labeled) are removed, as shown in Fig. 2c, the line segment between $x_{1^{\prime}}$ and $x_{2^{\prime}}$ (a convex combination of these two points) is a face of convex polyhedron $\operatorname{conv}\left(\left\{x_{1^{\prime}}, x_{2^{\prime}}, x_{3^{\prime}}, x_{4^{\prime}}, x_{5^{\prime}}\right\}\right)$ and $x_{i}$ lies on this face. Based on Theorem 1, the nonzero entries of the solution of Problem (9) must correspond to points $x_{1^{\prime}}$ and $x_{2^{\prime}}$. Indeed, their original points $x_{1}$ and $x_{2}$ lie in tangent space $T_{x_{i}}$. Then, the points from the tangent space of $M_{2}$ at $x_{i}$ are found out using Problem (9), even though the two manifolds are very close to each other. 


\subsection{Non-negative representation learning algorithm}

Problem (9) can be solved by

$$
\left\{\begin{array}{l}
\min _{c_{i}} \frac{1}{2}\left\|X_{i} c_{i}\right\|_{2}^{2} \\
\text { subject to } \mathbf{1}^{T} c_{i}=1 \text { and } c_{i} \geq 0 .
\end{array}\right.
$$

Theorem 2 states that the nonnegative condition $\lambda_{i} \geq 0$ can be replaced by $\|\lambda\|_{1} \leq 1$ under the condition $\mathbf{1}^{T} \lambda=1$. Hence, Problem (10) can be rewritten as:

$$
\left\{\begin{array}{l}
\min _{c_{i}} \gamma_{1}\left(\left\|c_{i}\right\|_{1}-1\right)+\frac{1}{2}\left\|X_{i} c_{i}\right\|_{2}^{2} \\
\text { subject to } \mathbf{1}^{T} c_{i}=1
\end{array}\right.
$$

where the parameter $\gamma_{1}$ controls the trade-off between the non-negativity of the solution and the affine reconstruction error. Point $x_{i}$ is a convex combination of all vertices $\left\{\frac{x_{j}-x_{i}}{\left\|x_{j}-x_{i}\right\|_{2}}\right\}_{j \neq i}$ of convex polyhedron $P$.

In order to obtain a new convex polyhedron from $P$, where $x_{i}$ lies on one of its faces and some points lying in tangent space $T_{x_{i}}$ are the vertices of this face, we must remove the points that are furthest away from $x_{i}$. To this end, the term $\left\|q_{i} c_{i}\right\|_{1}$ is added into Problem (11)

$$
\left\{\begin{array}{l}
\min _{c_{i}} \gamma\left\|q_{i} c_{i}\right\|_{1}+\gamma_{1}\left(\left\|c_{i}\right\|_{1}-1\right)+\frac{1}{2}\left\|X_{i} c_{i}\right\|_{2}^{2} \\
\text { subject to } \mathbf{1}^{T} c_{i}=1
\end{array}\right.
$$

where $q_{i} \triangleq\left[\frac{\left\|x_{1}-x_{i}\right\|_{2}}{\sum_{t \neq i}\left\|x_{t}-x_{i}\right\|_{2}} \cdots \frac{\left\|x_{N}-x_{i}\right\|_{2}}{\sum_{t \neq i}\left\|x_{t}-x_{i}\right\|_{2}}\right] \in \mathcal{R}^{1 \times N-1}$. Note that each element of $q_{i}$ is proportional to the distance between $x_{i}$ and the other points to penalize the furthest points. That is, the points closer to $x_{i}$ receive a lower penalty than those far away. To simplify the calculation, we use $\left\|q_{i} c_{i}\right\|_{1}$ rather than $\left\|q_{i} c_{i}\right\|_{2}$ because $\left\|c_{i}\right\|_{1}$ is already present in Problem (11). The parameter $\gamma$ controls the distance penalty and can be tuned. Ideally, the points sufficiently far away are assigned zero weight (or zerocontribution to $x_{i}$ ).

Here the original points corresponding to the nonzero entries of the solution $c_{i}$ of Problem (12) are more likely to be from the tangent space at the given query point. At the same time, every element of $c_{i}$ reflects the distance information. Then, the solutions for all data points can be used to build a similarity graph, on which a spectral clustering and embedding algorithm can be used. A more detailed description of how to use $c_{i}$ for clustering and embedding is given in Elhamifar and Vidal (2011). We call this method the non-negative representation learning algorithm (NRLA).

SMCE (Elhamifar and Vidal 2011) is similar to NRLA to automatically find out the neighbors for manifold clustering and embedding. Its optimization problem is

$$
\left\{\begin{array}{l}
\min _{c_{i}}\left\|Q_{i} c_{i}\right\|_{1} \\
\text { subject to }\left\|X_{i} c_{i}\right\|_{2} \leqslant \varepsilon \text { and } \mathbf{1}^{T} c_{i}=1
\end{array}\right.
$$

where $X_{i} \triangleq\left[\frac{x_{1}-x_{i}}{\left\|x_{1}-x_{i}\right\|_{2}} \cdots \frac{x_{N}-x_{i}}{\left\|x_{N}-x_{i}\right\|_{2}}\right] \in \mathcal{R}^{D \times N-1}$, and the diagonal elements of $Q_{i}$ are set to be $\frac{\left\|x_{j}-x_{i}\right\|_{2}}{\sum_{t \neq i}\left\|x_{t}-x_{i}\right\|_{2}} \in(0,1]$ to favor points that are close to $x_{i}$. The nonzero elements of the solution $c_{i} \in \mathcal{R}^{N-1 \times 1}$ are expected to indicate the points that are on the same manifold as $x_{i}$. However, Problem (13) does not guarantee the non-negativity of the nonzero entries of the solution. Thus, it cannot be analyzed using convex polyhedral theory. 


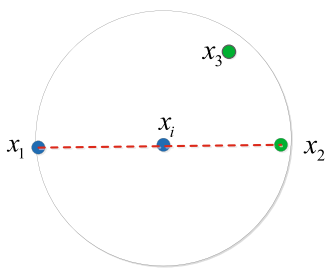

(a)

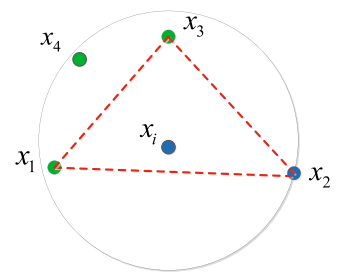

(b)

Fig. 3 Illustrate that the neighbors selected by NRLA preserve the local structure better than by traditional kNN. a An example where two neighbors of $x_{i}$ are selected. Traditional kNN selects the green points $x_{2}$ and $x_{3}$. NRLA selects the points $x_{1}$ and $x_{2}$. b An example where three neighbors of $x_{i}$ are selected. Traditional kNN selects the green points $x_{1}, x_{3}$ and $x_{4}$. NRLA selects the points $x_{1}, x_{2}$ and $x_{3}$

Moreover, the applications of SMCE are restricted, because we do not have the corresponding theoretical guarantees. As we will show in our experimental section, NRLA performs much better than SMCE.

The neighbors selected by NRLA preserve the local manifold structure better than traditional $\mathrm{kNN}$, when they come from the same tangent space. To illustrate this, we consider two examples shown in Fig. 3a, b, where all points come from the same tangent space. In the example of Fig. 3a with $k=2$, traditional kNN selects the green points $x_{2}$ and $x_{3}$ as the two nearest points. NRLA with two nonzero entries [in the solution of Problem (12)] selects the points $x_{1}$ and $x_{2}$. In the example shown in Fig. 3b, three neighbors are selected. Traditional kNN selects the green points $x_{1}, x_{3}$ and $x_{4}$ as the three nearest points. NRLA with three nonzero entries selects the points $x_{1}, x_{2}$ and $x_{3}$. Obviously, the local manifold structure is better preserved by the points selected by our method than those by traditional kNN.

We conduct several experiments to compare these two methods on synthetic and real data sets, which we discuss in next section. The experimental results verify that NRLA preserves the local manifold structure better than traditional $\mathrm{kNN}$. The reader may wonder why? According to the optimization problem in (12), we know that $x_{i}$ is a convex combination of the selected points, where both the distance and affine reconstruction are taken into account. The points selected by NRLA are near to $x_{i}$, and are almost uniformly distributed around $x_{i}$. Therefor, NRLA is better at preserving the local structure than traditional kNN, which is just based on the pairwise Euclidean distance.

The neighbors selected by our method are more likely to come from the tangent space of the manifold at the given point and well preserve the local manifold structure, which guarantee the performance of NRLA for simultaneous manifold clustering and embedding.

\subsection{Non-negative representation learning algorithm for $k$ neighbors selection}

At present, there are many methods for clustering and embedding such as Laplacian eigenmaps (LEM) (Belkin and Niyogi 2002) and LLE (Roweis and Saul 2000). Note that the first step of these methods is to select the neighbors. Traditional kNN is only based on the pairwise Euclidean distance, and the value of $k$ has a crucial influence on the performance of these algorithms. The neighbors selected by NRLA are more likely to be from the tangent space of the manifold at the given point and well preserve the local manifold structure, but this method is used for simultaneous clustering and embedding on multiple manifolds rather than selecting neighbors. Moreover, it is obvious that clustering errors lead to embedding errors. In fact, the number of the neighbors required by NRLA for clustering is different from 
for embedding. Our previous theoretical analysis shows that the clustering performance is related to the number of nonzero entries of the solution $c_{i}$ to the optimization problem in (12). This is because that retaining a small number points in NRLA is more likely to select the neighbors from the tangent space of the manifold at the given point. However, embedding requires more neighbors to well preserve the local structure.

Moreover, the solution (weights) of the optimization problem in (12) is ambiguous. The term $\left\|q_{i} c_{i}\right\|_{1}$ is used to ensure that every entry $c_{i j}$ of solution $c_{i}$ is inversely proportional to the distance term $q_{i j}$, which is related to the original data $\left\{x_{j}\right\}$. However, $X_{i}$ of the term $\left\|X_{i} c_{i}\right\|_{2}^{2}$ is related to the normalized vectors $\left\{\frac{x_{j}-x_{i}}{\left\|x_{j}-x_{i}\right\|_{2}}\right\}_{j \neq i}$, but not the original data $\left\{x_{j}\right\}$. The weights $c_{i}$ are ambiguous, which restricts the application of NRLA.

From these findings, we propose a new method for selecting $k$ neighbors. Based on NRLA, a new parameter $k$ is introduced into to Problem (12) for controlling the number of nonzero entries in the solution to a fixed value. The new optimization problem is

$$
\left\{\begin{array}{l}
\min _{c_{i}}\left\|q_{i} c_{i}\right\|_{1} \\
\text { subject to }\left\|X_{i} c_{i}\right\|_{2} \leqslant \varepsilon, \mathbf{1}^{T} c_{i}=1,\left\|c_{i}\right\|_{1} \leq 1,\left\|c_{i}\right\|_{0}=k,
\end{array}\right.
$$

where

$$
\left\{\begin{array}{l}
X_{i} \triangleq\left[\frac{x_{1}-x_{i}}{\left\|x_{1}-x_{i}\right\|_{2}} \cdots \frac{x_{N}-x_{i}}{\left\|x_{N}-x_{i}\right\|_{2}}\right] \in \mathcal{R}^{D \times N-1}, \\
q_{i} \triangleq\left[\frac{\left\|x_{1}-x_{i}\right\|_{2}}{\sum_{t \neq i}\left\|x_{t}-x_{i}\right\|_{2}} \cdots \frac{\left\|x_{N}-x_{i}\right\|_{2}}{\sum_{t \neq i}\left\|x_{t}-x_{i}\right\|_{2}}\right] \in \mathcal{R}^{1 \times N-1},
\end{array}\right.
$$

$c_{i} \in \mathcal{R}^{N-1 \times 1} .\|\cdot\|_{0}$ is the $\ell^{0}$ norm, which counts the nonzero entries of the solution vector $c_{i}$. The constraint term $\left\|c_{i}\right\|_{0}=k$ is introduced to fix the number of nonzero entries of the solution vector $c_{i}$ to $k$, where the meaning of the parameter $k$ is the same with the $k$ in traditional kNN. Here the indices of the $k$ nonzero elements of $c_{i}$ correspond to the $k$ selected neighbors of $x_{i}$. We call this method the non-negative representation learning algorithm for $k$ neighbors selection (NRLA-kNS). It is similar to traditional $\mathrm{kNN}$ that our method can be used within other clustering methods such as LEM or embedding methods such as LLE. Our previous analysis shows that if NRLA-kNS is used for clustering, the parameter $k$ should have a lower value; if NRLA-kNS is used for embedding, $k$ should have a higher value. The appropriate $k$ value can be obtained using the semi-supervised method. All these are shown in our experiments.

Another optimization problem that is related to (14) by the method of Lagrange multipliers is

$$
\left\{\begin{array}{l}
\min _{c_{i}} \gamma\left\|q_{i} c_{i}\right\|_{1}+\gamma_{1}\left(\left\|c_{i}\right\|_{1}-1\right)+\frac{1}{2}\left\|X_{i} c_{i}\right\|_{2}^{2} \\
\text { subject to } \mathbf{1}^{T} c_{i}=1,\left\|c_{i}\right\|_{0}=k
\end{array}\right.
$$

where the parameter $\gamma$ penalizes the distance, and $\gamma_{1}$ penalizes the nonnegativity. Our method is less sensitive to these two parameters since a new parameter $k$ is introduced. We have performed the related experiments, which are not present in this paper.

The optimization problem in (15) can be solved using the ALM method (Lin et al. 2010). For the reason that will become clear shortly, we introduce an additional vector variable $z_{i} \in \mathcal{R}^{N-1 \times 1}$ and solve the following optimization problem:

$$
\left\{\begin{array}{l}
\min _{c_{i}, z_{i}} \gamma\left\|q_{i} c_{i}\right\|_{1}+\gamma_{1}\left(\left\|c_{i}\right\|_{1}-1\right)+\frac{1}{2}\left\|X_{i} z_{i}\right\|_{2}^{2} \\
\text { subject to } \mathbf{1}^{T} z_{i}=1, z_{i}=c_{i},\left\|c_{i}\right\|_{0}=k
\end{array}\right.
$$


The complete algorithm for solving Problem (16) using ALM is shown in Algorithm 1.

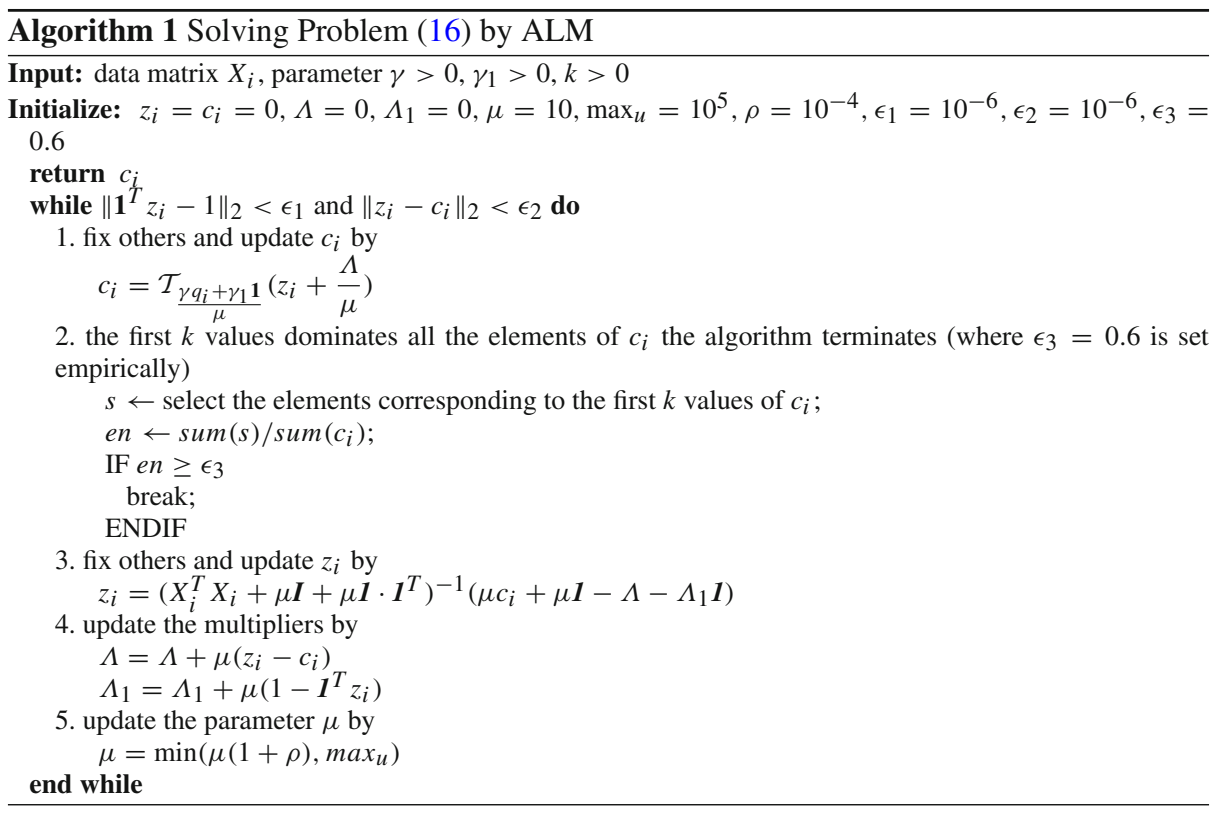

The aim of our method is to select the $k$ neighbors. Step 2 of the while loop of Algorithm 1 is designed so that when the sum of the first $k$ values dominates all the elements of $c_{i}$ the algorithm terminates (where $\epsilon_{3}=0.6$ is set empirically), rather than continuing until violating the while statement conditions. This strategy greatly increases the computing efficiency of NRLA-kNS. It's exciting to see that, in our experimental results, this method greatly improves the performance of embedding methods such as LLE and clustering methods such as LEM, when compared with traditional kNN. This is because NRLA-kNS takes into account both the distance and the geometrical structure, which leads it to select neighbors that almost always come from the tangent space of the manifold at the given point and well preserve the local structure.

We have also designed a NRLA algorithm on the basis of Algorithm 1, which retains the first $k$ values of $c_{i}$ and converts the others to 0. Experimental results show that NRLA is much more efficient and is better at simultaneous clustering and embedding than SMCE, even though a new parameter $k$ is introduced.

\section{Experiments and discussion}

We know that NRLA-kNS can be used for selecting neighbors, and the results can be used in clustering or embedding methods. NRLA can be used for simultaneous clustering and embedding. In this section, NRLA-kNS and NRLA are evaluated on synthetic data and realworld problems. All the experiments are carried out using MATLAB on a $2.40 \mathrm{GHz}$ machine with 2 GB RAM.

We evaluate the clustering performance using the average correct clustering rate. It is difficult to evaluate the embedding performance. At present, there are many quality assessments 


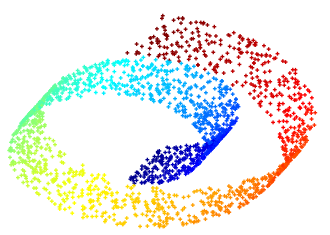

(a)

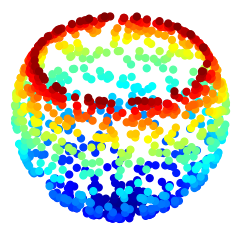

(b)

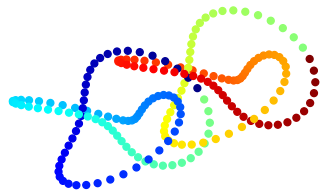

(c)

Fig. 4 Three examples of original synthetic data used in our experiments. a Swiss-roll manifold. b 2-sphere manifold. c Trefoil-knots manifold

for evaluating the performance of nonlinear dimensionality reduction (NLDR) methods. They can be split into two types: local and global quality assessments for NLDR. The local method (Lee and Verleysen 2008; Chen and Buja 2009; Valencia-Aguirre et al. 2009; Goldberg and Ritov 2009) mainly focuses on evaluating the local-neighborhood-preserving performance of the NLDR method. The global method (Tenenbaum et al. 2000; Dollár et al. 2007; Meng et al. 2011) measures the global-structure-holding capability of the technique.

In this paper, we use local continuity meta-criterion (LCMC) (Chen and Buja 2009). By computing the average overlap between the $\mathrm{kNN}$ neighboring set of the original data set and that of the representational set, the LCMC criterion is used as a diagnostic tool to measure the local structure of the learned embedding. Here $\mathrm{kNN}$ represents the $k$ nearest neighbors of a data set. Let the original data set be $X=\left\{x_{i}\right\}_{i=1}^{N}$ and the corresponding representational set obtained by the NLDR method be $Y=\left\{y_{i}\right\}_{i=1}^{N}$. The LCMC assessment is defined as the average local overlap, and is given by

$$
M_{L C}^{\prime}=\frac{1}{k^{\prime} N} \sum_{i=1}^{N}\left|\mathcal{N}_{k}\left(x_{i}\right) \bigcap \mathcal{N}_{k}\left(y_{i}\right)\right|,
$$

where $k^{\prime}$ is a pre-specified parameter that represents the overlap size, $\mathcal{N}_{k}\left(x_{i}\right)$ is the index set of $x_{i}$ 's $\mathrm{kNN}$, and $\mathcal{N}_{k}\left(y_{i}\right)$ is the index set of $y_{i}$ 's kNN (Zhang et al. 2012). A good quality embedding results in a high assessment value. In our experiments, $M_{L C}=1-M_{L C}^{\prime}$ is used.

\subsection{NRLA-kNS performance evaluation}

Neighbors selection is a very important step in manifold embedding methods such as LLE (Saul and Roweis 2003) and clustering methods such as LEM (Belkin and Niyogi 2002). Traditional $\mathrm{kNN}$ is a common method for selecting neighbors. In this section, we compare NRLA-kNS with traditional kNN in LLE and LEM. The experiments showed that NRLAkNS is less sensitive to parameters $\gamma$ and $\gamma_{1}$. We use the values of 10 and 1 for $\gamma$ and $\gamma_{1}$ when they are not specified.

\subsubsection{LLE with NRLA-kNS}

We first compare the two neighbors selection methods in LLE, which attempts to discover nonlinear structures in high dimensional data by exploiting the local symmetries of linear reconstructions (Saul and Roweis 2000). In these experiments, we use LLE to embed the swiss-roll manifold with the parameter equation $x^{1}=\cos u^{1}, x^{2}=u^{2}, x^{3}=u^{3} \sin u^{3}$, shown in Fig. 4a. The neighbors selection process of LLE is carried out by traditional kNN 


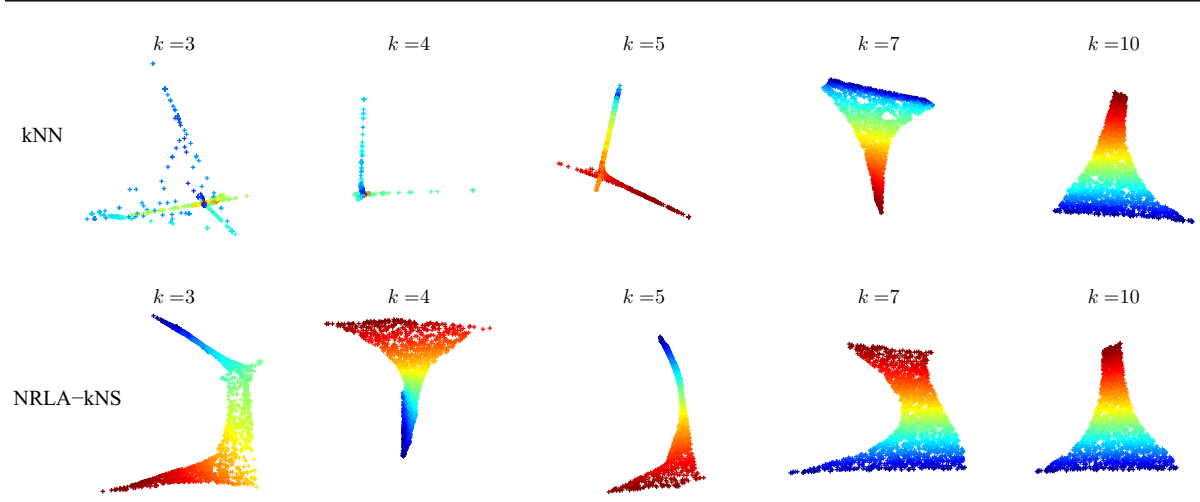

Fig. 5 Embedding results of the swiss-roll manifold obtained by LLE for different values of the parameter $k$, the $k$ neighbors are selected by traditional kNN or NRLA-kNS

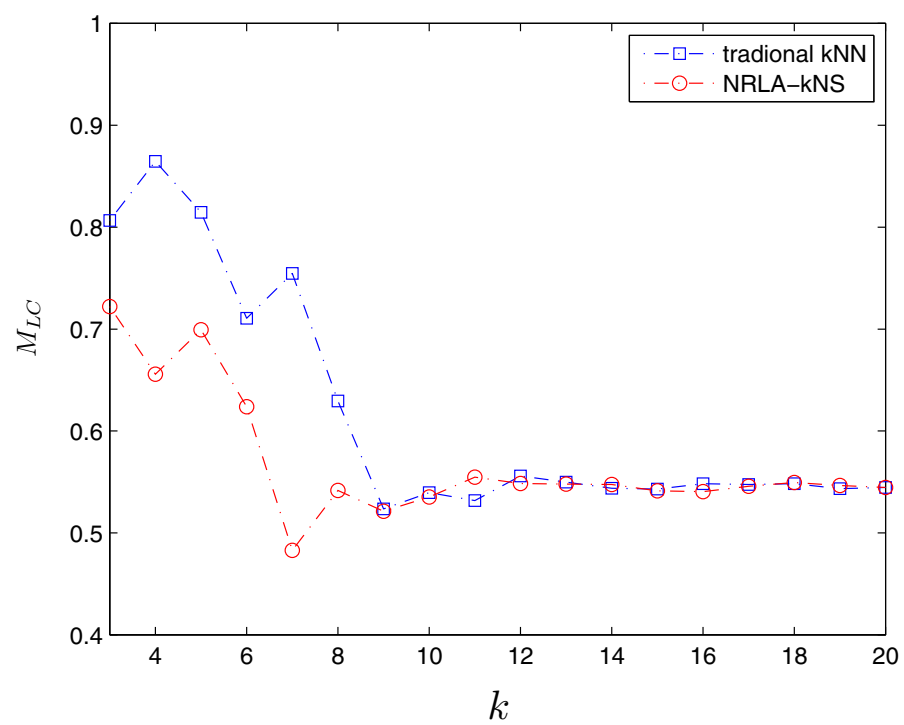

Fig. $6 M_{L C}$ assessments of the embedding results of the swiss-roll manifold obtained by LLE for different values of $k$, where the $k$ neighbors are selected by traditional kNN or NRLA-kNS

and NRLA-kNS, with $k \in[3,20]$. Different values of parameter $k$ have a significant influence on the performance of LLE using these two selection methods.

Figure 5 shows the embedding results of LLE, for $k \in\{3,4,5,7,10\}$. Figure 6 shows the $M_{L C}$ assessments of the embedding results, for $k \in[3,20]$. These results indicate that, for both methods, higher values of $k$ (or more neighbors) preserve the local structure better than lower values. Controlling the sparsity using NRLA-kNS or traditional $\mathrm{kNN}$ decreases the performance of LLE. Note that the results of LLE using our proposed method for a lower $k(k \in[3,6])$ are much better than the results using the traditional $\mathrm{kNN}$ method. This is because the neighbors picked by our method contain much more geometric information than the distance, which preserve the local structure better than the traditional technique. As $k$ increases, the performances using the two different methods become similar. 
Table 1 Clustering error rates on the trefoil-knots manifold using LEM for different values of $k$, where the $k$ neighbors are selected by NRLA-kNS and traditional kNN

\begin{tabular}{lrrrlll}
\hline$k$ & $2(\%)$ & $3(\%)$ & $4(\%)$ & $6(\%)$ & $7(\%)$ & $8(\%)$ \\
\hline NRLA-kNS & 0.0 & 0.0 & 11.5 & 13.5 & 13.5 & 49 \\
traditional kNN & 11.5 & 12.5 & 14.5 & 20.5 & 41 & 40.5 \\
\hline
\end{tabular}

\subsubsection{LEM with NRLA-kNS}

Next, we compare the two methods in LEM for clustering on synthetic and real data sets. The synthetic data set that we use is a trefoil-knots manifold (shown in Fig. 4c). In this example, the two knots are embedded in $\mathcal{R}^{100}$, corrupted with small Gaussian white noise. The data points are sampled such that among the two nearest neighbors, $1 \%$ of the data points are from the other manifold. Among the three and five nearest neighbors, there are 9 and $18 \%$ of data points from the other manifold, respectively (Elhamifar and Vidal 2011). We use LEM to cluster the data, selecting the neighbors using traditional $\mathrm{kNN}$ and NRLA-kNS for $k \in[3,8]$. The results are shown in Table 1 .

From this table, we can see that for the two neighbors selection methods the different values of $k$ have a significant influence on the performance of LEM. As $k$ increased, more neighbors are selected from the other manifold and then the performance is decreased. Note that the best performance is obtained by our NRLA-kNS when the value of $k$ is two or three. That is because that controlling the sparsity of the neighbors in NRLA-kNS (or giving a lower value of $k$ ) leads to picking neighbors that are more likely to come from the tangent space of the manifold at the given query point, which greatly improves the performance of LEM. However, traditional $\mathrm{kNN}$ is only based on the pairwise Euclidean distance, and thus only selects nearby points. Therefor, the performance of LEM using this neighbors selection method is much worse in this challenging example, regardless of the value of $k$.

The real data set that we use is a set of face images from the Extended Yale B database (Lee et al. 2005). Each subject has 64 images of $192 \times 168$ pixels, captured under a fixed pose and expression, with varying illuminations. In our experiments, two random faces are chosen and the average accuracy rates of the clustering using LEM for $k \in[2,15]$ are calculated, where the $k$ neighbors are selected by traditional kNN and NRLA-kNS, respectively.

The results are shown in Fig. 7. We can see that the average performance of NRLA-kNS is much better than traditional $\mathrm{kNN}$ for each $k \in[2,15]$. These results indicate that NRLA-kNS considers much more geometric information than the distance, which greatly increases the distinctions between two manifolds. Note that the results of our method for $k=2$ is perfect. As $k$ increased, the average accuracy rate of the clustering decreases using NRLA-kNS, and the result is close to that of traditional $\mathrm{kNN}$. This is because higher values of $k$ result in more neighbors being selected from the other subject. Controlling the sparsity in NRLAkNS improves the clustering performance. In our experiments, the best value of $k(=2)$ may be related to the intrinsic dimension of the data set.

\subsubsection{Simultaneous manifold clustering and embedding with NRLA-kNS}

Finally, we conduct experiments to evaluate simultaneous clustering and embedding on two manifolds. The left plots in Figs. 8 and 9 show the joint embeddings of two random faces from the Extended Yale B database (Lee et al. 2005) using (NRLA-kNS)+LEM and (traditional 


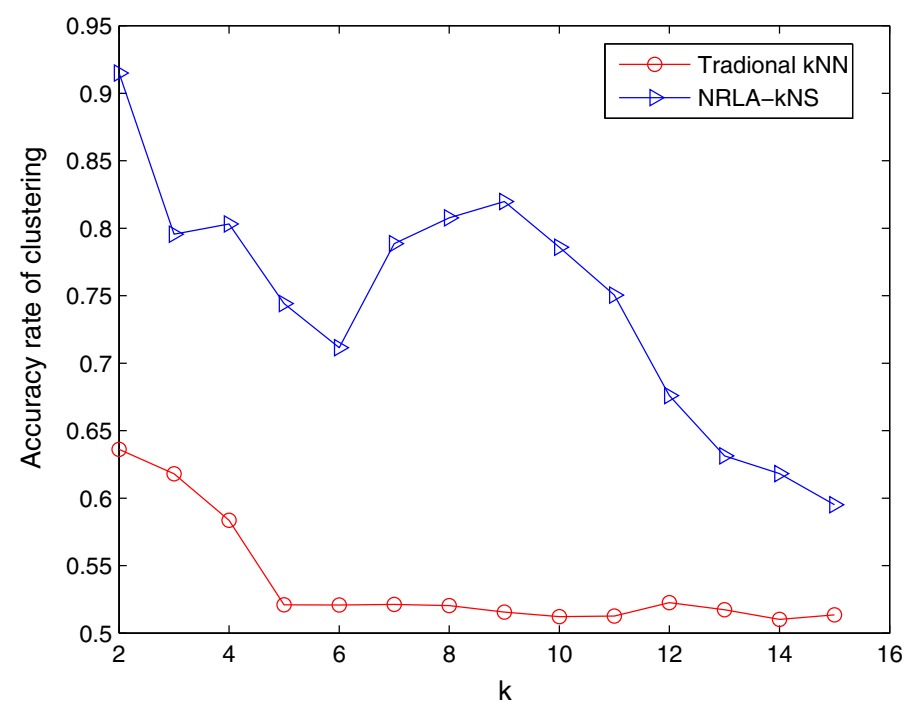

Fig. 7 Average accuracy rate of the clustering on two random faces in the Extended Yale B database using LEM for different values of $k$, where the $k$ neighbors are selected by traditional kNN and NRLA-kNS

$\mathrm{kNN})+\mathrm{LEM}$, respectively, where $k=2$. From these two plots, we can see that all the faces are well separated by our method, but (traditional kNN)+LEM does not perform well. This is because NRLA-kNs considers much more geometric information, which leads it to select points that are almost always from the correct manifold. The manifold clustering performance is greatly improved by replacing traditional $\mathrm{kNN}$ with NRLA-kNS. Figures $8 \mathrm{~b}, \mathrm{c}$ and $9 \mathrm{~b}, \mathrm{c}$ respectively show the embedding obtained by (NRLA-kNS)+LEM and (traditional $\mathrm{kNN}$ )+LEM for each cluster. Note that the direction of the light source in each cluster in Fig. 8 is obvious, but it does not exist in Fig. 9. This means that the neighbors selected by NRLA-kNS preserve the local structure of a manifold much better than the results by traditional kNN. In addition, embedding error faces can be seen in each cluster of Fig. 9. They are induced by the clustering errors.

\subsection{NRLA performance evaluation}

Both SMCE and our NRLA can be used for embedding in a manifold, clustering, or simultaneous clustering and embedding in multi-manifolds. In this section, we compare and evaluate the performance of these two methods on synthetic and real data sets.

The optimization problem for SMCE in Elhamifar and Vidal (2011) is

$$
\left\{\begin{array}{l}
\min _{c_{i}} \gamma\left\|Q_{i} c_{i}\right\|_{1}+\frac{1}{2}\left\|X_{i} c_{i}\right\|_{2} \\
\text { subject to } \mathbf{1}^{T} c_{i}=1,
\end{array}\right.
$$

where $X_{i} \triangleq\left[\frac{x_{1}-x_{i}}{\left\|x_{1}-x_{i}\right\|_{2}} \cdots \frac{x_{N}-x_{i}}{\left\|x_{N}-x_{i}\right\|_{2}}\right] \in \mathcal{R}^{D \times N-1}$, and the diagonal elements of $Q_{i}$ are set to $\frac{\left\|x_{j}-x_{i}\right\|_{2}}{\sum_{t \neq i}\left\|x_{t}-x_{i}\right\|_{2}} \in(0,1]$ to favor points that are close to $x_{i}$. The author believes that the clustering and embedding results obtained by SMCE are stable for $\gamma \in[1,200]$. However, the influences of $\gamma$ on the performance of this method are not evaluated. 


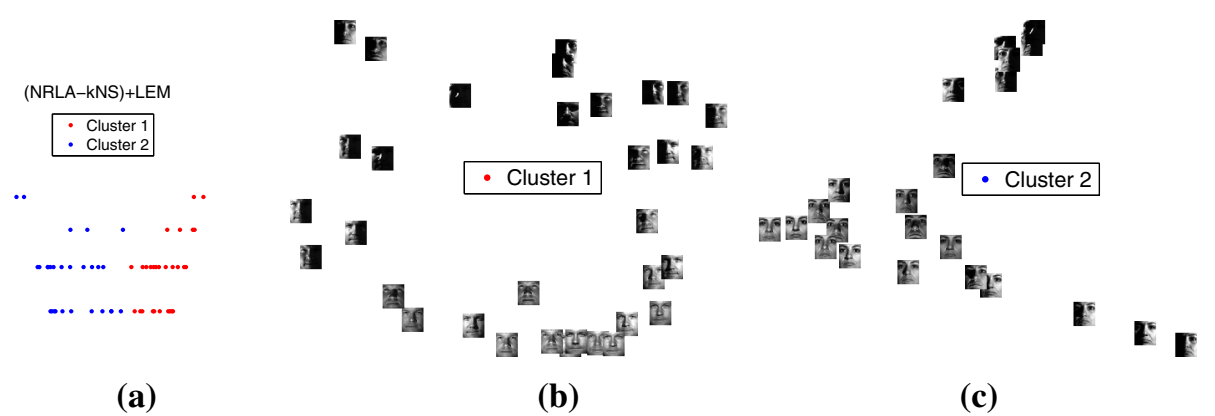

Fig. 8 Clustering and embedding of two faces by (NRLA-kNS)+LEM. a 2-D embedding. b (NRLAkNS)+LEM embedding for clustering 1. c (NRLA-kNS)+LEM embedding for clustering 2

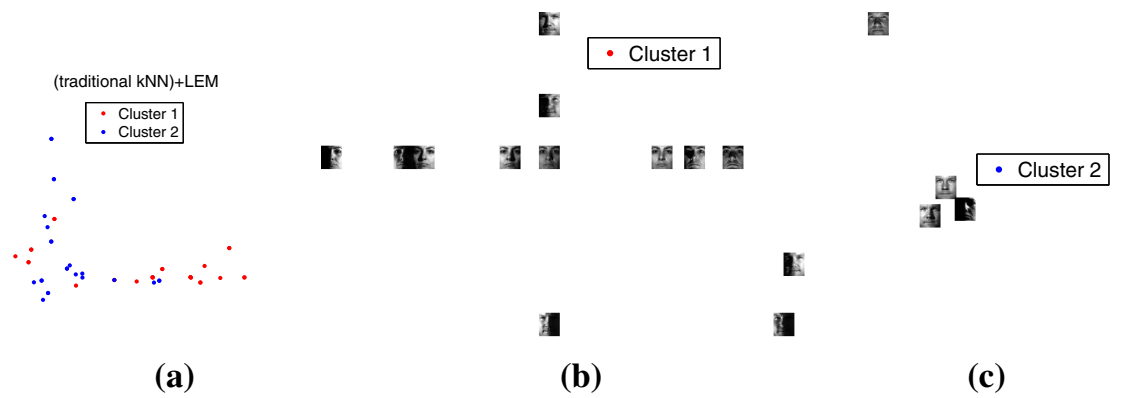

Fig. 9 Clustering and embedding of two faces by (traditional kNN)+LEM. a 2-D embedding. b (traditional $\mathrm{kNN}$ )+LEM embedding for clustering 1. c (traditional $\mathrm{kNN})+\mathrm{LEM}$ embedding for clustering 2

SMCE
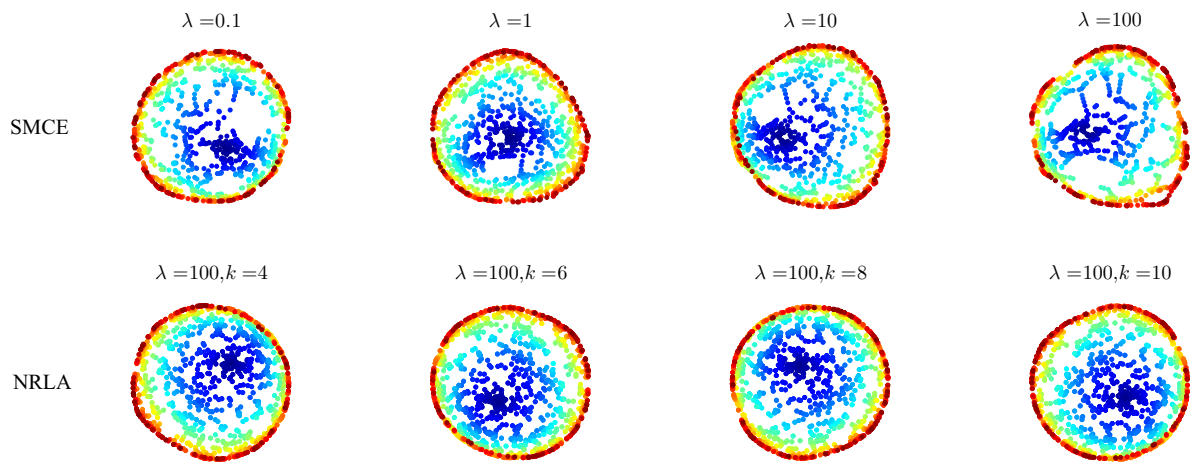

Fig. 10 Top embedding results of the 2-sphere manifold obtained by SMCE for different values of $\gamma$. Bottom embedding results obtained by our NRLA for different values of $k$

\subsubsection{NRLA applied to manifold embedding}

We first apply SMCE and NRLA to manifold embedding. The synthetic data set that we use is a 2-sphere manifold with a neighborhood of its north pole excluded, shown in Fig. 4b. Similar to the process in Elhamifar and Vidal (2011), we embed the data in $\mathcal{R}^{100}$ and add small Gaussian white noise to it. For $\gamma \in\{0.1,1,10,100\}$, the embedding results 


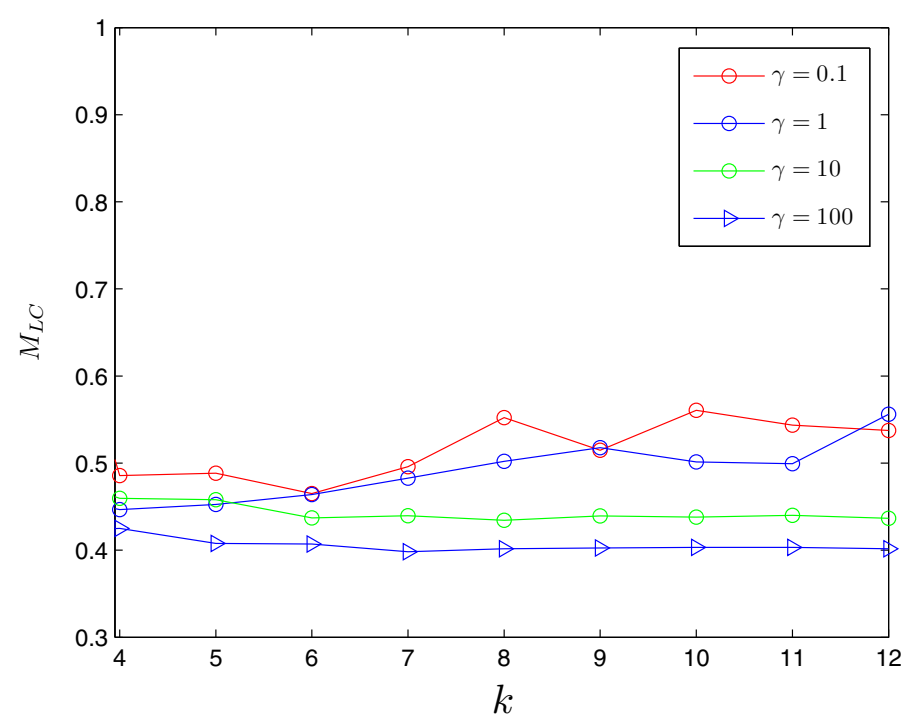

Fig. $11 M_{L C}$ assessments of the embedding results of the 2-sphere manifold obtained by NRLA for different values of $k$ to $\gamma$

by SMCE in a two dimensional Euclidean space are shown at the top of Fig. 10. From this figure, we can see that the parameter $\gamma$ in Problem (18) has a direct influence on the performance of the embedding. As explained in Elhamifar and Vidal (2011), very large values of $\gamma$ degrade the performance of the embedding, because the sparsity of the solution has more emphasis. Thus, $\gamma=100$ is the worst situation in the set $\{0.1,1,10,100\}$. Compared with SMCE, NRLA has a new parameter $k$. We apply NRLA for manifold embedding with $\gamma \in\{0.1,1,10,100\}$ and $k \in[4,12]$. Some of the embedding results for a two dimensional Euclidean space are shown at the bottom of Fig. 10, where $k \in\{4,6,8,10\}$ and $\gamma=100$ (the worst situation in the SMCE experiments). The embedding using NRLA performs better than using SMCE, even though the sparsity of the solution has more emphasis $(\gamma=100)$. Figure 11 shows the LCMC assessments of the embedding using NRLA for different values of $\gamma$ and $k$. Note that its performance is stable for both $\gamma$ and $k$.

\subsubsection{NRLA applied to manifold clustering}

Next, we apply SMCE and NRLA to manifold clustering. Table 2 shows the clustering error rates, average size of the selected neighbors, and computation time for different values of $\gamma$, where SMCE and NRLA are used on the trefoil-knots manifold (Elhamifar and Vidal 2011), shown in Fig. 4c. This is a challenging example for the two manifolds are close to each other. The table shows that the best clustering performance is obtained by NRLA, and the results are stable for different values of $\gamma$. The performance of SMCE is crucially influenced by this parameter. However, the author in Elhamifar and Vidal (2011) dose not discuss how to find the optimal parameter, which limits the application of SMCE. Note that NRLA takes significantly less time when compared with SMCE even though a new parameter $k$ is introduced. This is because of the particular design of Step 2 in Algorithm 1. In these experiments, the value of $k$ is fixed at 2 . 
Table 2 The clustering error rates, average size of the selected neighbors, and computation time (seconds) using SMCE, NRLA and SSC on the trefoil-knots manifold, where the results of SMCE and NRLA are to different values of $\gamma$
Table 3 Average accuracy rate of the clustering, average size of the selected neighbors and average computation time (seconds) using SMCE and NRLA on two random faces in the Extended Yale B face and ORL face datasets in 20 experiments with $\gamma=10$

\begin{tabular}{lllllll}
\hline$\gamma$ & 0.1 & 1 & 10 & 50 & 100 & 200 \\
\hline SMCE & & & & & & \\
Error rate(\%) & 14.5 & 10.5 & 13.5 & 0 & 0 & 0 \\
Neighbors & 51.56 & 20.5 & 3.35 & 2 & 2 & 2 \\
Time & 39.15 & 39.15 & 43.4 & 40.56 & 37.53 & 37.97 \\
NRLA & & & & & & \\
Error rate (\%) & 0.0 & 0.0 & 0.0 & 0.0 & 0.0 & 0.0 \\
Neighbors & 2 & 2 & 2 & 2 & 2 & 2 \\
Time & 111.72 & 33.88 & 4.03 & 1.62 & 2.0 & 1.4 \\
SSC & & & & & & \\
Error rate $(\%)$ & 47.0 & & & & & \\
Neighbors & 7.86 & & & & & \\
Time & 1.8 & & & & & \\
\hline
\end{tabular}

SMCE

$\begin{array}{ll}\text { Aver-Accuracy rate } & 79 \% \\ \text { Aver-neighbors } & 3.88 \\ \text { Aver-Time } & 47.96 \\ N R L A & \\ \text { Aver-Accuracy rate } & 92 \% \\ \text { Aver-neighbors } & 2 \\ \text { Aver-Time } & 24.14\end{array}$

\subsubsection{NRLA applied to simultaneous manifold clustering and embedding}

Finally, we apply SMCE and NRLA to simultaneous manifold clustering and embedding on almost 2592-dimensional vectors, which are formed by randomly selecting two faces from the Extended Yale B database (Lee et al. 2005). Table 3 shows the average accuracy rate of the clustering, average size of the selected neighbors and average computation time when SMCE and NRLA are used on two random faces in 20 experiments with $\gamma=10$. The average accuracy rate of the clustering is $79 \%$ for SMCE and $92 \%$ for NRLA. Our method has a better performance in the clustering than SMCE. Note that NRLA is almost $100 \%$ faster than SMCE. In these experiments, the average size of the neighbors is 3.88 by SMCE and 2 by NRLA (where 2 is set by the parameter $k=2$, an experimental value). Figures 12a and 13a respectively show the joint embeddings of two random faces by NRLA and SMCE. We can see that all the faces are reasonably well separated into their classes by our method, but SMCE does not perform well. That means the neighbors selected by NRLA are more likely to be from the correct manifold than by SMCE, even though a new parameter $k$ is introduced in our method. Figures $12 \mathrm{~b}, \mathrm{c}$ and $13 \mathrm{~b}$, c show the embedding obtained by LEM for each cluster. The direction of the light source can be seen in the two figures, but there are embedding error faces in every cluster of Fig. 13. They are induced by the clustering errors of SMCE.

At the same time, we use SSC (Elhamifar and Vidal 2009) for clustering on the trefoil-knots manifold. It's clustering error rate is $47 \%$ (shown in Table 2). Moreover, we apply NRLA, 


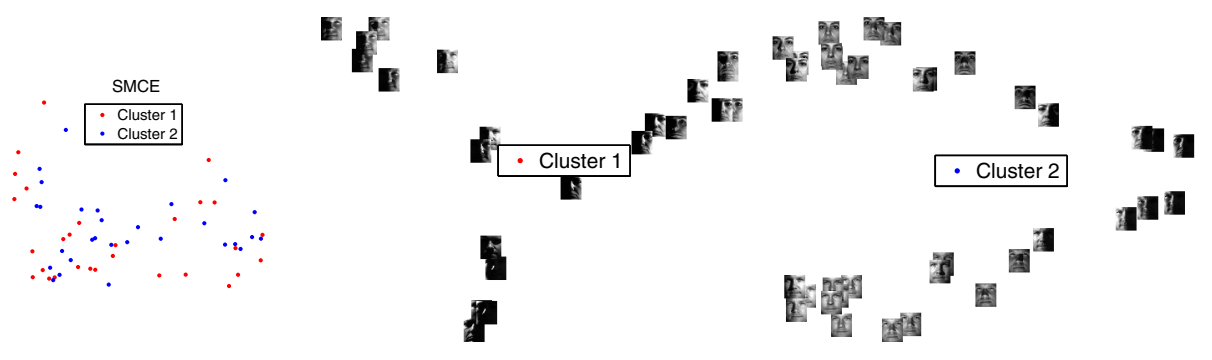

(a)

(b)

(c)

Fig. 12 Clustering and embedding of two faces by SMCE. a 2-D embedding. b SMCE embedding for clustering 1. c SMCE embedding for clustering 2

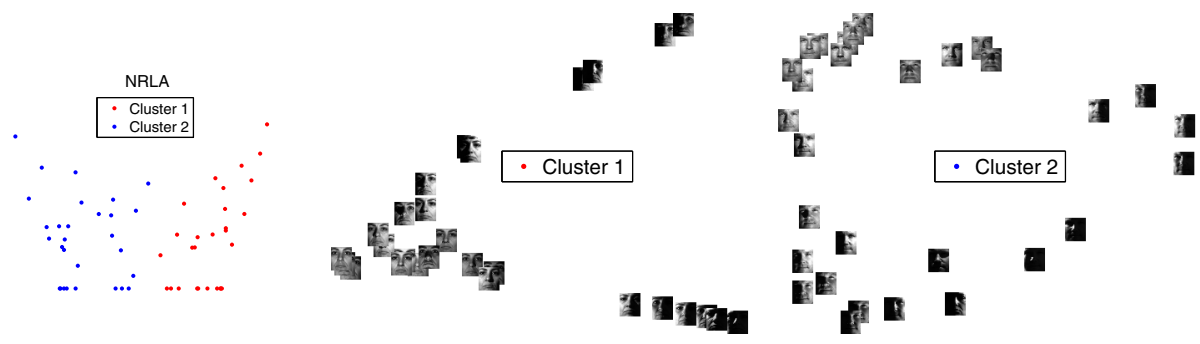

(a)

(b)

(c)

Fig. 13 Clustering and embedding of two faces by NRLA. a 2-D embedding. b NRLA embedding for clustering 1. $\mathbf{c}$ NRLA embedding for clustering 2
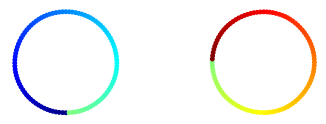

NRLA

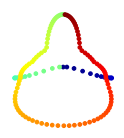

SMCE
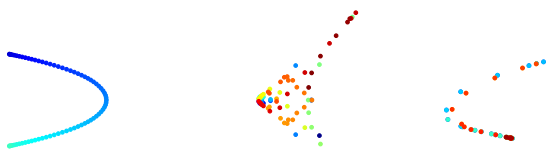

$\mathrm{SSC}$

Fig. 14 The results for simultaneous clustering and embedding using NRLA, SMCE (with $\gamma=10$ ) and SSC on the trefoil-knots manifold

SMCE and SSC to simultaneous manifold clustering and embedding on this synthetic data set. The results are shown in Fig. 14.

\subsection{Discussion}

The furthest points are removed by the distance term $q_{i}$. However, in some cases, this mechanism does not always choose local neighbors from the tangent space of the manifold at the given query point to be on a face. To illustrate this, consider the three manifold pieces shown in Fig. 15a, where the red centered point and its nearest points are labeled. If the furthest points (not labeled) are removed, the remaining points $\left\{x_{j^{\prime}}(j=1,2, \ldots, 8)\right\}$ form a convex polyhedron, as shown in Fig. 15b. In this case, $\operatorname{conv}\left(\left\{x_{4^{\prime}}, x_{8^{\prime}}\right\}\right)$ is not a face of convex polyhedron $\operatorname{conv}\left(\left\{x_{j^{\prime}}(j=1,2, \ldots, 8)\right\}\right)$, and the optimization problem in (16) cannot select the points lying in tangent space $T_{x_{i}}$. If the other far away points $x_{1^{\prime}}, x_{3^{\prime}}, x_{4^{\prime}}, x_{5^{\prime}}, x_{7^{\prime}}, x_{8^{\prime}}$ are 


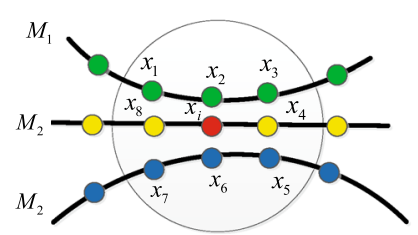

(a)

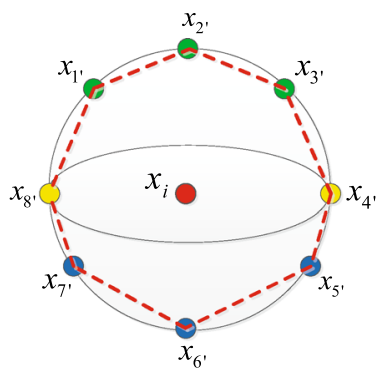

(b)

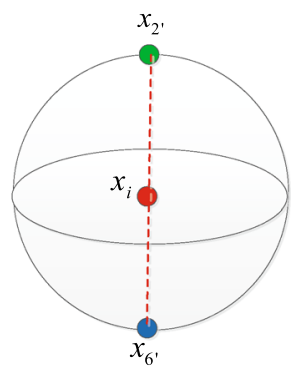

(c)

Fig. 15 Example that NRLA-kNS can not select local neighbors from the tangent space at the given query point to be on a face. a The original data in the three manifold pieces, where the red centered point and its nearest points are labeled. b After normalizing all points and removing the furthest points (not labeled), $x_{i}$ is not on a face. c After removing points $x_{1^{\prime}}, x_{3^{\prime}}, x_{4^{\prime}}, x_{5^{\prime}}, x_{7^{\prime}}, x_{8^{\prime}}$, the remaining points $x_{2^{\prime}}$ and $x_{6^{\prime}}$ do not lie in tangent space $T_{x_{i}}$

all removed, as shown in Fig. 15c, the remaining points $x_{2^{\prime}}$ and $x_{6^{\prime}}$ form a face of a convex polyhedron. The optimization problem in (16) would select the points $x_{2}$ and $x_{6}$, which do not lie in tangent space $T_{x_{i}}$. In this case, our method cannot be used for exact clustering.

\section{Conclusions}

In this paper, we have proposed a novel neighbors selection algorithm based on convex polytopes called NRLA-kNS. The neighbors selected by our method are almost always from the tangent space of the manifold at the given query point and well preserve the local manifold structure. The results can be used in manifold clustering methods such as LEM, or embedding methods such as LLE. We compared our method with the widely used traditional $\mathrm{kNN}$ technique and found that NRLA-kNS is much better for both LEM and LLE. Moreover, we replaced the non-negative condition by $\|.\|_{1}$ when obtaining our representation. We demonstrated the effectiveness and efficiency of the proposed method using experiments on synthetic data and a real-world problem.

Acknowledgments This work was supported by National Science Foundation of China under Grants 61375065 and 61432014, partially supported by National Program on Key Basic Research Project (973 Program) under Grant 2011CB302201.

\section{References}

Belkin, M., \& Niyogi, P. (2002). Laplacian eigenmaps and spectral techniques for embedding and clustering. In T. G. Dietterich, S. Becker, \& Z. Ghahramani (Eds.), Advances in Neural Information Processing Systems 14 (pp. 585-591). MIT Press.

Chen, L., \& Buja, A. (2009). Local multidimensional scaling for nonlinear dimension reduction, graph drawing, and proximity analysis. Journal of the American Statistical Association, 104(485), 209-219.

Cheng, J., Yi, Z., \& Zhou, J. (2010). Subspace learning of neural networks. Boca Raton, FL: CRC Press Inc.

Dollár, P., Rabaud, V., \& Belongie, S. (2007). Non-isometric manifold learning: Analysis and an algorithm. In Proceedings of the 24th international conference on machine learning (pp. 241-248). ACM.

Elhamifar, E., \& Vidal, R. (2009). Sparse subspace clustering. In Computer vision and pattern recognition (CVPR) 2009. IEEE Conference on (pp. 2790-2797). IEEE. 
Elhamifar, E., \& Vidal, R. (2011). Sparse manifold clustering and embedding. In J. Shawe-Taylor, R. S. Zemel, P. L. Bartlett, F. Pereira, \& K. Q. Weinberger (Eds.), Advances in neural information processing systems 24 (pp. 55-63). Curran Associates, Inc.

Ferrandiz, S., \& Boullé, M. (2010). Bayesian instance selection for the nearest neighbor rule. Machine Learning, 81(3), 229-256.

Gallier, J. (2011). Basics of affine geometry. In S. Antman, P. Holmes, L. Sirovich, \& K. R. Sreenivasan (Eds.), Geometric methods and applications (pp. 7-63). Berlin: Springer.

Goh, A., \& Vidal, R. (2007). Segmenting motions of different types by unsupervised manifold clustering. In Computer vision and pattern recognition, 2007. CVPR'07. IEEE Conference on (pp. 1-6). IEEE.

Goh, A., \& Vidal, R. (2008). Clustering and dimensionality reduction on riemannian manifolds. In Computer vision and pattern recognition CVPR 2008. IEEE Conference on (pp. 1-7. IEEE). IEEE.

Goldberg, Y., \& Ritov, Y. (2009). Local procrustes for manifold embedding: A measure of embedding quality and embedding algorithms. Machine Learning, 77(1), 1-25.

Grunbaum, B., Klee, V., Perles, M. A., \& Shephard, G. C. (1967). Convex polytopes. Berlin: Springer.

Henk, M., Richter-Gebert, J., \& Ziegler, G. M. (1997). Basic properties of convex polytopes. In J. E. Goodman, \& J. O’. Rourke (Eds.), Handbook of discrete and computational geometry (pp. 243-270). Boca Raton, FL: CRC Press, Inc.

JIANG, T., CHEN, Xl, ZHANG, Yf, \& XIONG, Zy. (2009). Improved knn using clustering algorithm [j]. Computer Engineering and Applications, 7, 048.

Lee, J. A., \& Verleysen, M. (2008). Rank-based quality assessment of nonlinear dimensionality reduction. In M. Verleysen (Ed.), 16th European symposium on artificial neural networks (ESANN 2008) (pp. 49-54).

Lee, K. C., Ho, J., \& Kriegman, D. (2005). Acquiring linear subspaces for face recognition under variable lighting. Pattern Analysis and Machine Intelligence, IEEE Transactions on, 27(5), 684-698.

Li, X., Lv, J.C., \& Yi, Z. (2013). Manifold alignment based on sparse local structures of more corresponding pairs. In Proceedings of the twenty-third international joint conference on Artificial Intelligence (pp. 2862-2868). AAAI Press.

Lin, Z., Chen, M., \& Ma, Y. (2010). The augmented lagrange multiplier method for exact recovery of corrupted low-rank matrices. arXiv preprint arXiv:1009.5055.

Liu, G., Lin, Z., \& Yu, Y. (2010). Robust subspace segmentation by low-rank representation. In Proceedings of the 27th international conference on machine learning (ICML-10) (pp. 663-670).

Lv, J. C., Tan, K. K., Yi, Z., \& Huang, S. (2009). Convergence analysis of a class of hyvärinen-oja's ica learning algorithms with constant learning rates. Signal Processing, IEEE Transactions on, 57(5), 1811-1824.

Lv, J. C., Tan, K. K., Yi, Z., \& Huang, S. (2010). A family of fuzzy learning algorithms for robust principal component analysis neural networks. Fuzzy Systems, IEEE Transactions on, 18(1), 217-226.

Meng, D., Leung, Y., \& Xu, Z. (2011). A new quality assessment criterion for nonlinear dimensionality reduction. Neurocomputing, 74(6), 941-948.

Polito, M., \& Perona, P. (2002). Grouping and dimensionality reduction by locally linear embedding. In T. G. Dietterich, S. Becker, \& Z. Ghahramani (Eds.), Advances in neural information processing systems 14 (pp. 1255-1262). Cambridge, MA: MIT Press.

Roweis, S. T., \& Saul, L. K. (2000). Nonlinear dimensionality reduction by locally linear embedding. Science, 290(5500), 2323-2326.

Saul, L. K., \& Roweis, S. T. (2000). An introduction to locally linear embedding. Unpublished. http://www. cs.toronto.edu/ roweis/lle/publications.html.

Saul, L. K., \& Roweis, S. T. (2003). Think globally, fit locally: Unsupervised learning of low dimensional manifolds. The Journal of Machine Learning Research, 4, 119-155.

Tenenbaum, J. B., De Silva, V., \& Langford, J. C. (2000). A global geometric framework for nonlinear dimensionality reduction. Science, 290(5500), 2319-2323.

Tipping, M. E., \& Bishop, C. M. (1999). Mixtures of probabilistic principal component analyzers. Neural Computation, 11(2), 443-482.

Valencia-Aguirre, J., Álvarez-Mesa, A., Daza-Santacoloma, G., \& Castellanos-Domínguez, G. (2009). Automatic choice of the number of nearest neighbors in locally linear embedding. In E. Bayro-Corrochano \& J. O. Eklundh (Eds.), Progress in pattern recognition, image analysis, computer vision, and applications (pp. 77-84). Berlin: Springer.

Vidal, R., Ma, Y., \& Sastry, S. (2005). Generalized principal component analysis (gpca). Pattern Analysis and Machine Intelligence, IEEE Transactions on, 27(12), 1945-1959.

Zhang, P., Ren, Y., \& Zhang, B. (2012). A new embedding quality assessment method for manifold learning. Neurocomputing, 97, 251-266. 